\title{
High performance liquid chromatography: principles and clinical applications
}

\author{
Ian M Bird
}

Detection of disease often relies on identifying abnormal molecules or imbalanced ratios of normal molecules in body fluids, these being reflections of abnormal metabolism. The study of these molecules and their interactions in both healthy and diseased states aids diagnosis and the monitoring of treatment. To carry out such investigations we need to identify, quantify, and ultimately purify the molecules concerned.

One technique that is highly successful in achieving all three objectives is column chromatography, and particularly high performance liquid chromatography (HPLC).

\section{General principles of column chromatography}

The underlying principle of chromatography is that molecules not only dissolve in liquids but can also "dissolve" (absorb) on to or interact with the surface of solids. (Water will dissolve coffee, and coffee solution can also stain cloth.) If a molecule dissolved in a liquid is passed down a column of solid particles with which it interacts it will move more slowly than the solvent, spending some time dissolved in the liquid (liquid phase) and some on the solid (solid phase). As such molecules take longer to pass down the column than molecules that do not interact with the solid phase they emerge from the bottom of the column later (fig 1).

The separation of molecules by chromatography depends on exploiting the subtle differences in certain physical properties of the molecules in samples: their solubility in water, solubility in organic solvents, net

Department of

Biochemistry, University of Edinburgh, Edinburgh EH3 9YW

Ian M Bird, PHD, postdoctoral research fellow

Series edited by: Drs Peter and John Hayes.

BrMed f 1989;299:783-7
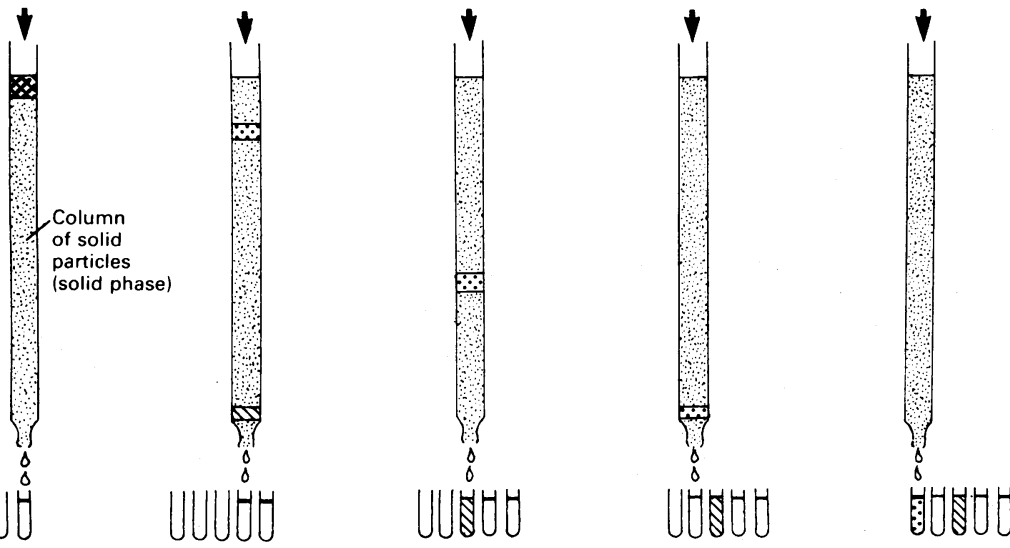

FIG 1 -Solvent $(\downarrow \downarrow$ ) containing sample molecules $A \mathbb{N}$ and $B \mathbb{\$}$ is continuously passed through a column. Solid material in the column can dissolve $B$ on its surface, but not $A$, so that $B$ spends part of its time dissolved on the solid surface, and moves more slowly down the column than $A$, which spends all of its time in the liquid

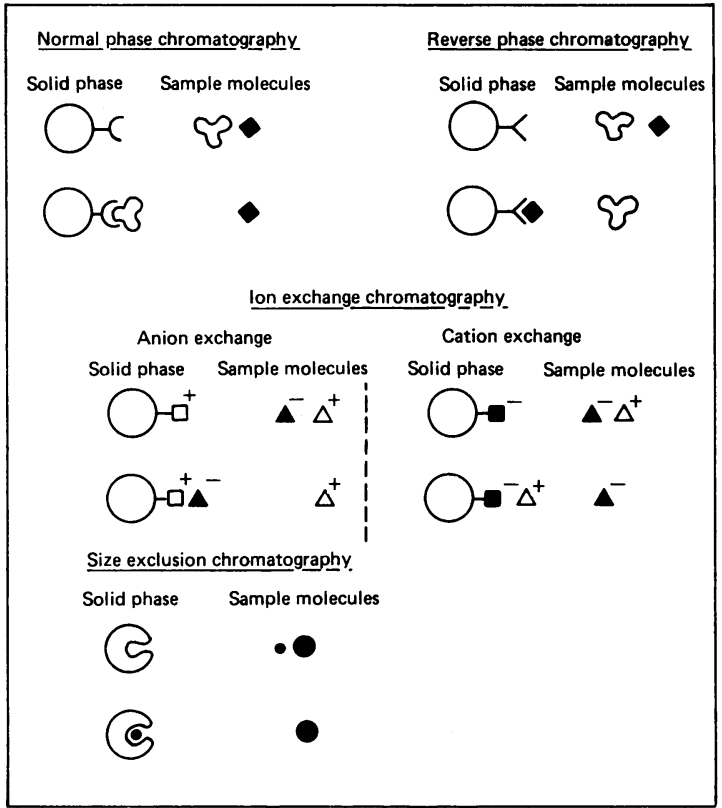

FIG 2-Various forms of high performance liquid chromatography (HPLC) are based on solid phases of silica particles (O). In normal phase HPLC hydrophilic binding groups $(-c)$ on the silica surface attract hydrophilic $(\wp)$ but not hydrophobic $(\diamond)$ molecules. In reverse phase HPLC hydrophobic binding groups $(\prec)$ on the silica surface attract hydrophobic ( ) but not hydrophilic ( $\checkmark)$ molecules. In ion exchange HPLC the functional group attached may express a positive $\left(-\square^{+}\right)$or negative $\left(-\mathbf{Q}^{-}\right)$charge and attract counter charged molecules $\left(\triangle^{-}, \Delta^{+}\right)$. In size exclusion $H P L C$ the silica particles have been modified to create pores in the surface ((S) to trap and retain small (•) but not large $(\mathbf{)}$ molecules

exchange chromatography it must be charged, and for size exclusion chromatography the solid phase must be able physically to retard smaller molecules as they move through the column.

Though the mechanism underlying separation will be decided by the choice of solid phase medium, the actual separation achieved by normal phase, reverse phase, and ion exchange chromatography will also be influenced by the choice of medium for the liquid phase, which competes with the solid phase for sample molecules. Thus in normal phase chromatography solvents of greater increasing polarity will more effectively remove polar molecules from the solid phase, and in reverse phase chromatography increasingly hydrophobic (organic) solvents will more readily remove non-polar molecules from the solid phase. (This is equal but opposite to the normal phase mechanism - hence the term reverse phase.) In ion exchange chromatography the affinity of charged molecules for the solid phase is reduced when buffers of increasing salinity or changing $\mathrm{pH}$, or both, are used to suppress ionisation.

Often a liquid phase of changing composition is used so that at the beginning of the method it is not as good at dissolving the molecules of interest as is the solid phase and consequently the molecules spend almost all their time on the solid phase. As the composition of the liquid phase changes, however, it becomes a better 
solvent for the molecules of interest and so they are increasingly removed from the solid phase into the liquid phase. This technique is called gradient elution and can be applied to normal phase, reverse phase, and

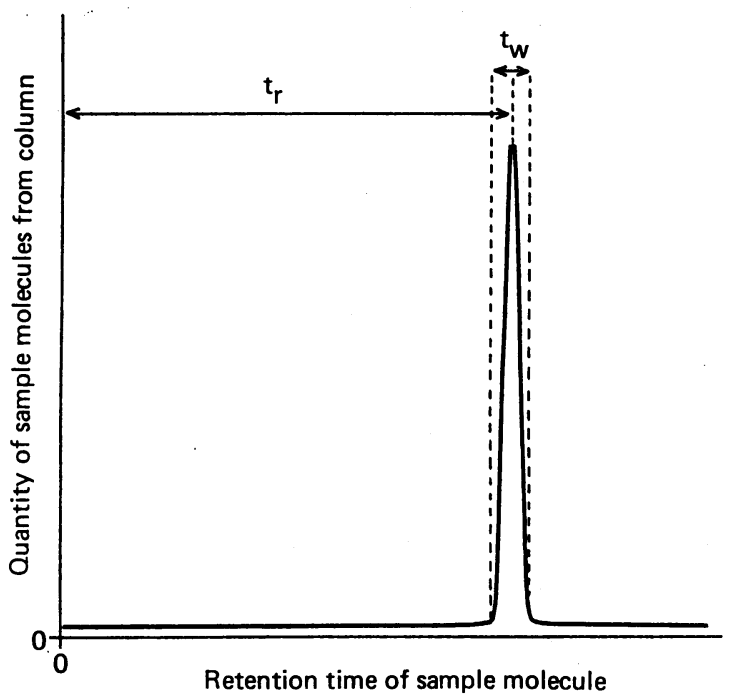

FIG 3-Calculation of efficiency of a column from the equation $n=16$ $\left(t_{r}\right)^{2} /\left(t_{w}\right)$. The retention time of the peak (tr) and time taken for the peak to emerge from the column (tw) can be measured from a chart recording
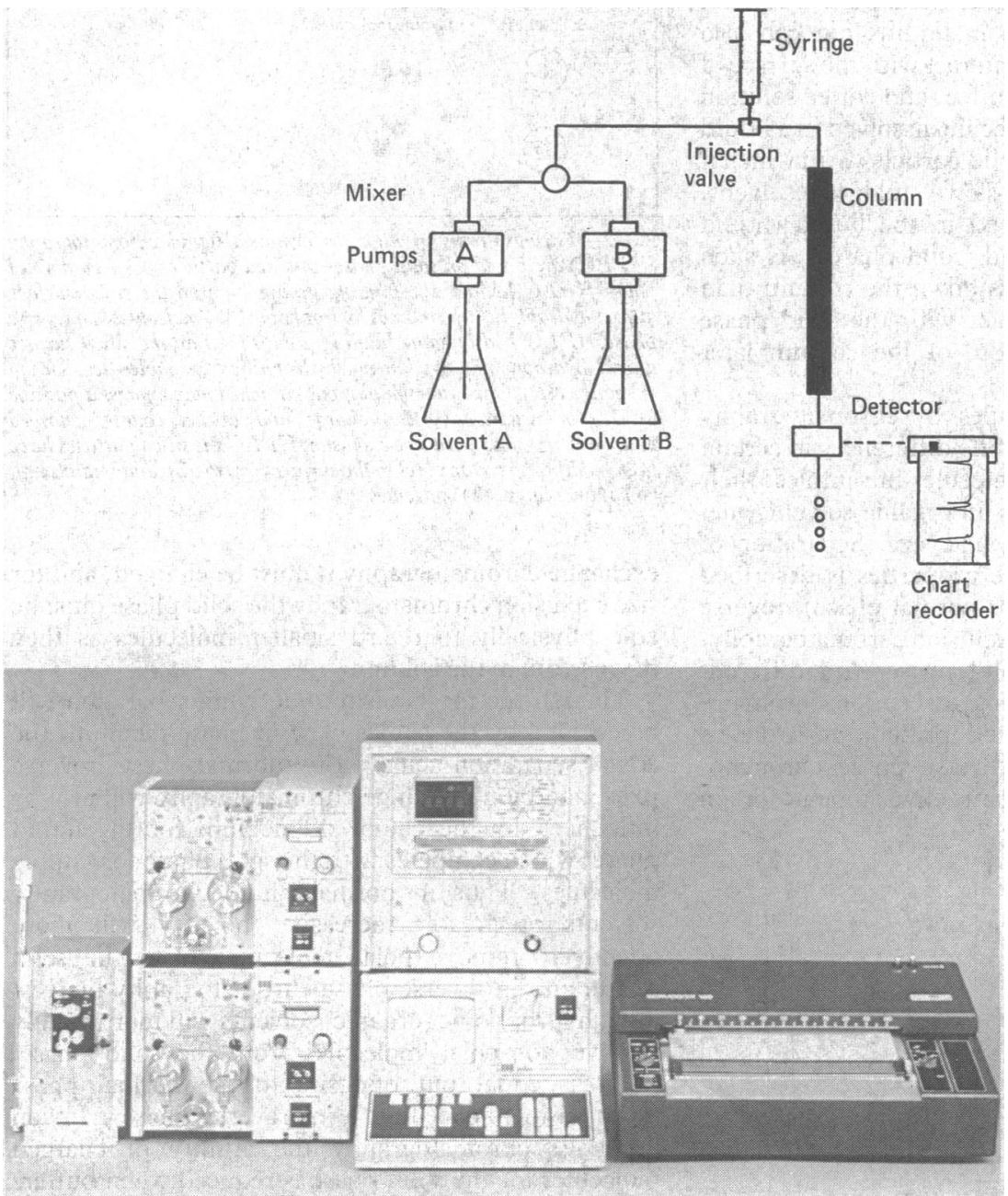

FIG 4-(Top) Owing to the high back pressure of HPLC columns solvent is delivered by a pump. If a gradient elution is needed then two (or more) pumps are used to deliver the different solvent components to the column through a mixing chamber. Samples are put in the flowing solvent by an injection valve. Molecules emerging from the column are monitored continuously by an in line detector and the result recorded on a chart recorder; (Bottom) A gradient HPLC system: HPLC column mounted on the side of a unit containing the injection valve (extreme left); two HPLC pumps (left of centre, top and bottom); mixer (on bottom pump); pump controller (centre bottom); ultraviolet detector (centre top); chart recorder (right) WATERS CHROMATOGRAPHY ion exchange column chromatography but not to size exclusion chromatography, which depends only on the size of the molecule.

High performance liquid chromatography columns

For any chromatography column to be maximally effective at retarding a sample molecule the column must be designed so that the sample molecule has ample opportunity to come into contact with the solid phase. One means of achieving this is simply to make the column bigger. Many columns may be a few centimetres long and have a volume $<10 \mathrm{ml}$, but some columns may be metres long and have a volume $>1$ litre. The improvements achieved by altering column dimensions, are however, limited. A much more successful approach is to make the particles of solid phase medium as small as possible. As the particle size gets smaller (generally from $250 \mu \mathrm{m}$ to $5 \mu \mathrm{m}$ ) the column performance improves for two reasons: a greater area of solid phase is exposed to the sample molecule (for any given column volume); and the volume of solvent (and so diffusion time) between the particles is reduced. Both these changes increase the chance that a sample molecule dissolved in the solvent will come into contact with, and so be retarded by, the solid phase. But reducing the particle size of the solid phase brings practical problems. As the particle size is reduced and the space between the particles decreases so the back pressure of the column increases. Thus many solid phases (for example, those based on cellulose or organic resins) that have good physicochemical properties at normal operating pressures $(<0.5 \mathrm{kPa})$ can never give "high performance" with reduced particle size: they would collapse at the high working pressures (up to $3.5 \mathrm{kPa}$ ).

Fortunately, there are some solid phases which can withstand these high operating pressures, and of these the most versatile and successful are those based on silica. Particles of silica $(5 \mu \mathrm{m}-10 \mu \mathrm{m})$ provide an excellent solid phase for high performance normal phase chromatography. Chemical modification of the surface provides materials which act as reverse phase and ion exchange media. Alternatively, physical modification of silica to create tiny pores give the solid phase required for the retardation of smaller molecules in size exclusion chromatography (fig 2). These basic types of modified silica can be used to separate most, if not all, molecules of interest. Thus it is mainly columns with solid phases derived from silica that form the basis of most high performance liquid chromatography (HPLC) methods.

The best indication of how much better high performance columns are than normal columns is given by determining column efficiencies from the expression: $\mathrm{n}=16\left(\mathrm{t}_{\mathrm{r}}^{2}\right) /\left(\mathrm{t}_{\mathrm{w}}\right)$

where $\mathrm{n}$ is a measure of efficiency, $\mathrm{t}_{\mathrm{r}}$ is the retention time for a given peak of sample molecules, and $t_{w}$ is the width of the peak (fig 3). Typical values of $n$ for normal columns would be $<500$ but for high performance columns of only a few millilitres volume the value for $\mathbf{n}$ can easily exceed 10000 .

\section{Equipment for high performance liquid chromatography}

The equipment used to operate HPLC columns can be divided into two main areas, the delivery of solvent and sample to the column and the detection of molecules emerging from the column. Figure 4 shows the equipment used for solvent and sample delivery. Close regulation of the flow rate of solvent through the column and of the composition of the solvent in gradient procedures is achieved by microprocessor feedback systems controlling the pumps. Together 
with the use of a sample injection valve this makes any HPLC method highly reproducible.

To identify the appearance of the various molecules being separated as they emerge from the column a detector is often attached "in line" with the column outlet. Usually detectors monitor the absorbance or fluorescence of the emerging molecules, but other methods may sometimes be used. In line monitoring enables an immediate and continuous record of the separation achieved to be recorded on paper by a chart recorder.

\section{Advantages and disadvantages of HPLC as a routine method}

An appropriate combination of column, solvent, and detector in HPLC will allow the separation of one type of molecule from others. Thus it can be used to rapidly establish a purification or assay method. In addition, it can be used to identify and simultaneously quantify many components of a complex mixture (such as mixtures of amino acids or steroids or drug metabolites), giving it a large advantage over radioimmunoassay, or equivalent techniques, in which separate assays would be required. Furthermore, HPLC allows unanticipated components in a sample to be detected, whereas in radioimmunoassay the assay is specific to only one (or a few) molecules.

HPLC does not lend itself readily to analysis of large numbers of samples (unlike radioimmunoassay). Few

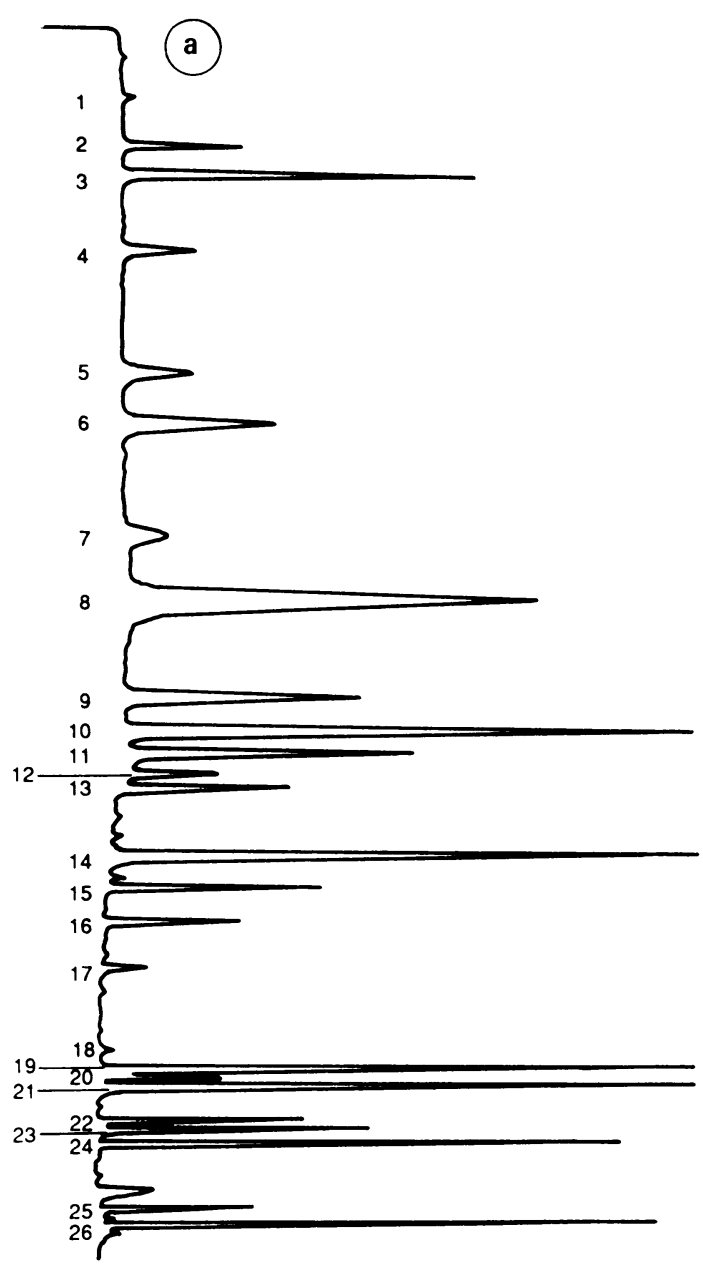

methods of HPLC elution can be carried out in less than half an hour for one sample, but if samples are limited in number a good throughput can be achieved with automated systems of sample injection. Many such systems are now commercially available. Though the initial cost for such automated apparatus may be high, the running costs are often low. A further disadvantage of HPLC is that, being a sensitive analytical technique, it is also sensitive to interference by sample or solvent contaminants and this may result in artefacts or in an apparently successful method being unusable in routine analysis. Using high purity solvents and prepreparing samples appropriately will overcome these difficulties in most cases. Successfully establishing a routine HPLC method will often depend on such considerations.

\section{Clinical applications}

HPLC has a considerable range of applications in both clinical research and routine clinical analysis. The following examples illustrate the variety of molecules commonly analysed by this method.

Measuring glycated haemoglobin, especially type lc, is a means of monitoring longer term plasma glucose control in diabetic patients. As all the la, b, and c forms of glycated haemoglobin are less positively charged than normal haemoglobin at neutral $\mathrm{pH}$, a method that separates proteins by charge should separate glycated from normal haemoglobin. Methods based on chroma-

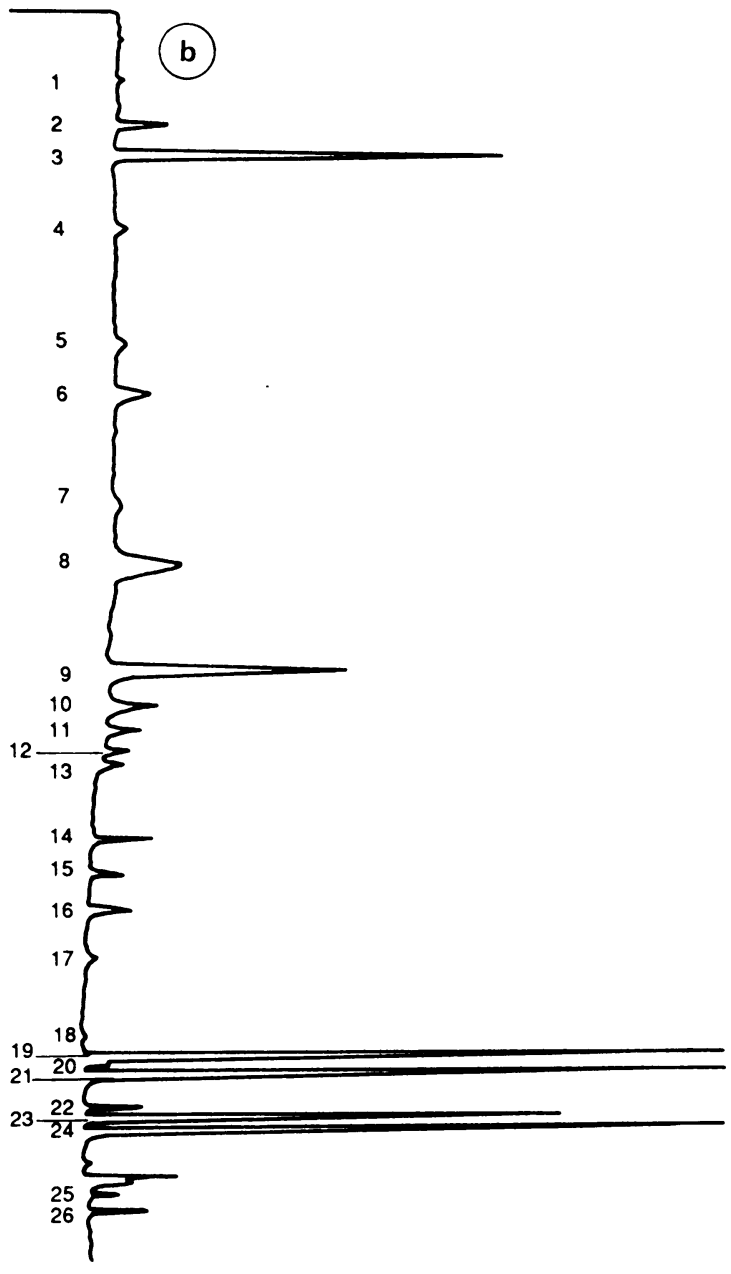

FIG 5-Reverse phase HPLC separation of plasma amino acids from normal and abnormal subjects after chemical modification of amino acids with orthophthalaldehyde. (Courtesy' of $G$ Blundell)

a. Amino acid profile obtained from undiluted normal plasma. Peaks 3, 9, and 21 are not naturally occurring but were added as standards

b. Amino acid profile obtained from diluted (fourfold) plasma from a subject with maple syrup urine disease. Peaks 3, 9, and 21 were added as standards. Note relatively high peaks 19,23, and 24 (valine, isoleucine, and leucine) compared with other amino acids

$1=$ aspartate, $2=$ glutamate, $3=$ homocysteic acid, $4=$ cysteine, $5=$ asparagine, $6=$ serine, $7=$ histidine, $8=$ glutamine, $9=$ homoserine, $10=$ glycine, $11=$ threonine, $12=$ citrulline, $13=$ arginine, $14=$ alanine, $15=$ taurine, $16=$ tyrosine, $17=\alpha$-amino- $n$-butyrate, $18=$ ethanolamine, $19=$ valine, $20=$ methionine, $21=$ norvaline, $22=$ phenylalanine, $23=$ isoleucine, $24=$ leucine, $25=$ ornithine, $26=$ lysine 
tography from small cation exchange columns as well as various electrophoresis methods have been used successfully to resolve glycated from non-glycated haemoglobins. A serious complication, however, is that other forms of haemoglobin (such as $\mathrm{HbF}, \mathrm{HbS}$, and $\mathrm{HbC}$ ) associated with haemoglobinopathies migrate with the glycated haemoglobins both in electrophoresis and on cation exchange columns. Cation exchange HPLC, however, can resolve all the subtypes of glycated haemoglobin both from each other and from the F, S, and C forms. This form of assay shows excellent precision with rapid separation, and many manufacturers market HPLC systems dedicated entirely to this purpose.'

The ability of HPLC to resolve closely related molecules makes it the method of choice for detailed investigation of many congenital metabolic disorders or diseases. For instance, though amino acids in plasma or urine, or both, can be investigated with paper chromatography, thin layer chromatography, or high voltage electrophoresis, these methods give relatively poor separation and results are difficult to quantify. Ion exchange HPLC methods have been much more successful in separating, identifying, and quantifying the main amino acid species in plasma and in urine. Chemical pretreatment of amino acid samples to increase their solubility in organic media has enabled 27 different amino acids to be resolved by reverse phase HPLC (fig 5). ${ }^{2}$ The relative value of such methods is shown in maple syrup urine disease and phenylketonuria. Maple syrup urine disease, which is caused by a deficiency of branched chain oxo-acid decarboxylases that results in an accumulation of leucine and often of isoleucine and valine, can be detected by paper or thin layer chromatography or electrophoresis. With raised concentrations of plasma phenylalanine, however, an amino acid profile is necesssary to find the underlying cause. Phenylalanine concentration alone may be raised, as in phenylketonuria, or may increase as a consequence of hypertyrosinaemia or owing to generalised hyperaminoacidaemia. A high resolution technique like HPLC can differentiate between these states with the same basic method.

Other general types of molecule can also be separated into individual smaller molecules by HPLC to give metabolic profile. With ion exchange HPLC techniques the biogenic amines can be analysed. This is of value in investigating conditions such as phaeochromocytoma (associated with raised catecholamines) or carcinoid syndrome (raised serotonin).

Abnormalities in purine or pyrimidine metabolism often result in abnormalities in levels of nucleotides. Simmonds et al recently described an anion exchange HPLC procedure for the separation of the major nucleotides and their corresponding deoxyderivatives. ${ }^{3}$ Investigation of the erythrocyte nucleotide profiles of subjects showed that characteristic changes were detectable in six out of eight purine or pyrimidine disorders tested. The method can also be used on fetal blood and so allows antenatal screening for disorders such as adenosine deaminase deficiency (fig 6), which leads to fetal immunodeficiency syndrome, as well as more benign disorders such as xanthine oxidase deficiency.

Reverse phase HPLC has been used successfully for identifying and quantifying individual urinary porphyrins. Thus it is possible, for instance, to distinguish between porphyria cutanea tarda (defective uroporphyrinogen decarboxylase), which results in highly carboxylated porphyrins, and porphyria variegata (deficiency of protoporphyrin oxidase), which results in moderately carboxylated porphyrins.

Many HPLC methods have been developed for the study of vitamins and their metabolites. It is possible to separate vitamin $\mathrm{D}$ from its 25-hydroxy and 1,25-

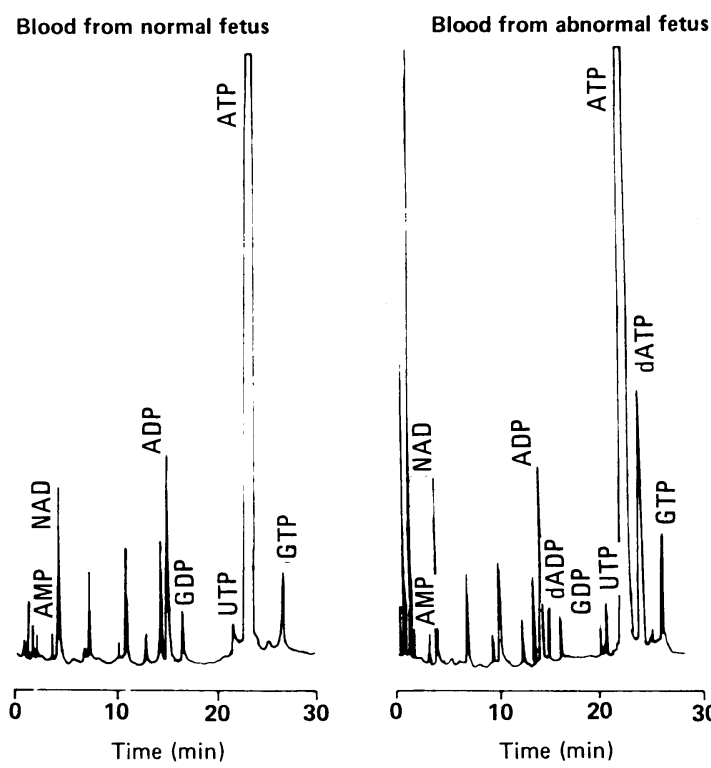

FIG 6-Nucleotides and deoxynucleotides from fetal erythrocyte hydrolysates of normal and abnormal (adenosine deaminase deficient) subjects. Note increase in deoxynucleotides $d A T P$ and $d A D P$ in the abnormal subject. Separation was achieved by gradient anion exchange HPLC. (Reproduced with permission from Simmonds et al ${ }^{3}$ )

dihydroxy forms and so investigate the possibility that reduced formation of the active dihydroxy form was due to impaired renal function or congenital abnormalities.

One of the main areas in which HPLC is used is in therapeutic drug monitoring. Monitoring is beneficial under a variety of circumstances - for example, when the therapeutic dose is close to the toxic dose, when signs of toxicity are difficult to detect clinically, when the rate of metabolism varies widely between patients, or when drug metabolism is impaired owing to organ dysfunction or altered by other drugs. Monitoring when rates of metabolism might vary is especially important if the drug metabolite is the therapeutically active form or the toxic form.

Drugs that are commonly monitored by HPLC include antiasthmatic drugs (theophylline and its active metabolities, including caffeine), anticonvulsants such as carbamazepine, phenobarbitone, phenytoin, ethosuximide, primidone and valproate, tricyclic antidepressants, and cardioactive drugs such as procainamide and propranolol. In many cases the drug may be monitored by radioimmunoassay, but when no antibody to the drug (or metabolite) exists, or if simultaneous measurement of a mixture of drugs or metabolites is required then HPLC is more effective. ${ }^{+}$

HPLC can readily be used to identify drug metabolites that may be important in drug toxicity. This is well illustrated by cyclosporin A, a powerful immunosuppressant that has revolutionised organ transplantation, but which has potentially serious nephrotoxic side effects. The original radioimmunoassay method showed a correlation between concentrations of cyclosporin A and nephrotoxicity that was not found by HPLC. This difference was due to the lack of specificity of the antibody used: radioimmunoassay measured not only cyclosporin A but also several of its metabolites, whereas HPLC clearly separated cyclosporin A from them. These differences imply that one or more of the metabolites of cyclosporin A may be responsible for nephrotoxicity, rather than cyclosporin A itself. Once the nephrotoxic metabolite has been identified by high resolution techniques such as HPLC then a specific radioimmunoassay can be developed.

HPLC is also widely used to identify causes of poisoning. Common toxins that are freely available include alcohols (ethanol, methanol, ethylene glycol), analgesics (aspirin, paracetamol), barbiturates, and 


\section{Glossary}

Adsorption (normal phase) HPLC - separation of molecules on the basis of their solubility in water

Chiral molecules - those containing a carbon atom to which are bound four different groups. As the arrangement of these groups is asymmetric the molecule may exist in two forms that are mirror images of each other yet have the same constituent parts

Gradient elution - a form of HPLC in which the composition of the liquid phase is altered with time, so encouraging the transfer of molecules from the solid phase to the liquid phase

Ion exchange HPLC - separation of molecules on the basis of their net charge

Liquid phase - the solvent passing through the HPLC column

Reverse phase HPLC - separation of molecules on the basis of their solubility in organic solvents

Size exclusion HPLC - separation of molecules on the basis of their size

Solid phase-the surface of the solid particles (in an HPLC column) with which molecules can interact

paraquat, all of which can be rapidly identified by HPLC, as can those substances associated with drug abuse (opiates, amphetamines, hallucinogens, and cannabinoids).

\section{HPLC and the future}

The practical limit to the efficiency of HPLC columns is set by the solid phase. Improvements in the efficiency of silica based columns are unlikely, as reducing solid phase particle size to $<5 \mu \mathrm{m}$ results in excessively high back pressures, so improvements need new types of solid phases. Recently, highly porous beads of organic resins to which functional groups can be attached have been successfully developed. These columns give similar separations to conventional columns but at much lower back pressures. Columns formed from stacks of cellulose fibre discs on to which functional groups are attached also give lower back pressures than conventional columns. The ability to pump columns at low pressure makes it possible in many cases to speed up the analysis by increasing flow rate. Alternatively, low pressure columns allow the use of pumps constructed of glass and plastic rather than steel and quartz, which is useful when separating enzymes that may be inhibited by heavy metals or when the buffers used attack steel. The increasing availability of such columns may ultimately result in the development of cheaper low pressure HPLC systems.

Alternative forms of silica based stationary phase are also being developed. These have chiral functional groups attached that are capable of selectively binding other chiral molecules. Many drugs are active in only one of their two possible chiral forms, so this development is of great importance clinically. These developments and others yet to come will ensure a role for HPLC in both research and routine clinical analysis.

\section{Conclusion}

HPLC is an extremely powerful means of separating molecules according to their solubility in water, solubility in organic solvents, net positive charge, net negative charge, or size. The great variety of columns and solvents available makes this method highly adaptable to the investigation of a wide range of molecules, but the equipment is costly and throughput of samples is poor compared with other methods. When no other method is available or a complex mixture of components requires simultaneous assay then the method is invaluable as a diagnostic tool and a monitor of treatment.

I thank Dr H A Simmonds and Dr G Blundell for providing the illustrations as indicated and Mrs E Ward for her patience and careful typing of the manuscript.

1 Goldstein DE, Little RR, Wiedmeyer H, England JD, McKenzie EM. Glycated hemoglobin: methodologies and clinical applications. Clin Chem 1986; 32:B64-70

2 Blundell G, Brydon WG. High performance liquid chromatography of plasma amino acids using orthophthalaldehyde derivatisation. Clin Chim Acto 1987; 170:79-84.

3 Simmods HA, Fairbanks LD, Morris GS, Webster DR, Harley EN. Altered erythrocyte nucleotide patterns are characteristic of inherited disorders of purine and pyrimidine metabolism. Clin Chim Acta 1988;171:197-210.

4 Matsumoto K, Kikuchi H, Kano S, Iri H, Takahashi H, Umino M. Automated determination of drugs in serum by liquid chromatography with column switching. I. Separation of anti-epileptic drugs and metabolites. Clin Chem 1988;34:141-4.

\section{BOOKS RECEIVED}

\section{Addiction \\ Stepping Out: Freedom from Minor Tranquillisers and Sleeping Pills. S Trickett. (Pp 47; £1.50 paperback.) 1988. Available from Broadcasting Support Services, PO Box 7, London W3 3 XJ. \\ Oncology \\ Cancer of the Bile Ducts and Pancreas. Ed P E Preece, A Cuschieri, R D Rosin. (Pp xiii + 328; figs; £52.) Philadelphia: Saunders, 1989. Distri- buted by Harcourt Brace Jovanovich. ISBN 0-7216-2631-9. \\ Clinical Ocular Oncologv. D H Char. Illustrated by J Weddell. ( $\mathrm{Pp} x+402$ figs; colour plates; $\{130$.) New York: Churchill Livingstone, 1989. ISBN 0-443-08569-2. \\ Ophthalmology \\ Diagnosis and Management of Orbital Tumors. J A Shields. (Pp xii + 401; figs; Tumors. J A Shields. (Pp xii + 401; figs;
£80.) Philadelphia: Saunders, 1989. Distributed by Harcourt Brace Jovan- ovich. ISBN 0-7216-2791-9. \\ Paediatrics \\ Caring for Children with Chronic Illness: Issues and Strategies. R E K Stein. (Pp}

$\mathrm{xxx}+301 ; \quad \$ 36.95$. .j New York: Springer, 1989. ISBN 0-8261-5900-1. Fighting, Teasing and Bullying: Simple and Effective Ways to Help Your Child. J Pearce. (Pp 96; figs; $£ 1.99$ paperback.) Wellingborough: Thorsons, 1989. ISBN 0-7225-1722-X.

Tantrums and Tempers: Tried-and Tested Ways of Helping Your Child Cope with Strong Emotions. J Pearce. Cope with Strong Emottons. J Pearce.
(Pp 96; figs; £l.99 paperback. Wellingborough: Thorsons, 1989. Wellingborough:
ISBN 0-7225-1721-1.

\section{Pharmacology}

ABPI Data Sheet Compendium 1989 90 Together with the Code of Practice for the Pharmaceutical Industry. Association of the British Pharmaceutica Industry. (Pp xvi+1854; $\quad$ I14. London: Datapharm Publication 1989. ISBN 0-907102-00-X.

South African Medicines Formulary. Ed E A Conradie, J L Straughan. (Pp E A Conradie, J L Straughan. ( $P$ p Xv1 + 407; paperback, price not stated. Pinelands: Medical Association of
South Africa, 1988. ISBN 0-86811South

Pharmacology - clinical

Drug Death: a Danger of Hospitalization. $\mathrm{R}$ P Hoffmann. ( $\mathrm{Pp} \times \mathbf{x}+131$
\$29.75.) Springfield: Thomas, 1989. ISBN 0-398-05554-8.

MCQs in Clinical Pharmacology. B Davies, A Sinclair, $S$ Jackson. ( $P$ 219; f6.50 paperback.) Edinburgh: Churchill Livingstone, 1989. ISBN 0-443-03729-9.

\section{Physiology}

Survival in Space: Medical Problems of Manned Spaceflight. R Harding. ( $\mathbf{P}$ $\mathrm{xix}+227$; figs; £14.95.) London: Routledge, 1989. ISBN 0-415-00253

\section{Psychiatry}

Current Reviews in Psychiatry. No 3 "Adolescent Psychiatry." P Hill. Series editors E S Paykel, H G Morgan. (Pp x+346; £29.95.) Edinburgh: Churchill Livingstone, 1989. ISBN 0-443-02774-9.

Self-Treatment for Phobias. K Sell. ( $\mathrm{Pp}$ 32; $£ 2.25$ each, or 10 copies for $£ 20$ 32; $£ 2.25$ each, or 10 copies for $£ 20$ paperback.) 1989, Phobic Action, Greater London House,

\section{Psychology}

Advances in Suicidology. Vol 1. "Suicide and Its Prevention: the Role of Attitude and Imitation." Ed R F W
Diekstra, R Maris, S Platt, et al. (Pp $\mathbf{x}+371 ; \quad \$ 70$ paperback.) Published Organisation. Leiden: Brill, 1989. ISBN 90-04-08613-7.

Against Therapy. Warming: Psychotherapy May be Hazardous to Your Menta Health. J Masson. (Pp 320; £15.) London: Collins, 1989. ISBN 0-00 215092-1. Childhood and Human Nature: the
Development of Personality. S Wolff.

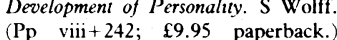
(Pp viii $+242 ; \quad £ 9.95$ paperback.)
London: Routledge, 1989. ISBN London: Rourted

Living with Tinnitus: Dealing with the Ringing in Your Ears. R Hallam. ( $\mathrm{Pp}$ 128; figs; $£ 4.99$ paperback.) Wellingborough: Thorsons, 1989. ISBN 0-7225-1801-3.

Longman Applied Psychology. "Health Psychology." P G Harvey. General editor P Feldman. ( $P p x+137$; figs; £4.95 paperback.) London: Longman, 1989. ISBN 0-582-29623-4.

\section{Rehabilitation}

Everyday Aids and Appliances Articles from the British Medical fournal. Ed G

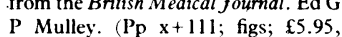
$\mathrm{P}$ Mulley. (Pp $\mathrm{x}+111$; figs; $£ 5.95$,
overseas $£ 7$ paperback, including posioverseas 27 paperback, including post-
$£ 5.45$, overseas $£ 6.50$ including postage.) London British Medical fourmal, 1989. In North America available from the American College of Physians, PO Box 7777-R-0270, Philate PA 19175, USA. ISBN 0-7279-024I-5. Functional Electrical Stimulation Standing and Walking After Spinal Cord Injury. A R Krali, T Bajd. (Pp vi + 198 figs; £66.50.) Florida: CRC Press, 1989. Distributed by Wolfe Medica Publications. ISBN 0-8493-4529-4.

Respiratory medicine

Asthma: Basic mechanisms and Therapeutic Perspectives. Ed J R Vane, G A Higgs, S A Marsico, G Nistico. (Pp xiv +252 ; figs; $\{40$.) Rome: Pythagora Press, 1989. ISBN 88-85852-01-7.

Statistics

Statistics with Confudence: Confidence Intervals and Statistical Guidelines. Ed M J Gardner, D G Altman. (Pp xviii + 140; figs; $£ 7.95$, overseas $£ 9.50$ paperback, including postage, airmail ove seas. BMA members 17.45 , overseas $£ 9$ including postage.) London: British Medical fournal, 1989. In North America available from the American America available from the American College of Physicians, PO Box 7777-R ISBN 0-7279-0222-9. 